(1)

${ }^{1}$ Centro Hospitalar de São João, Porto, Portugal.

2Faculdade de Medicina da Universidade do Porto, Porto, Portugal.

3|3S - Instituto de Investigação e Inovação em Saúde da Universidade do Porto, Porto, Portugal.

\title{
Pneumology and sleep disordered breathing training in Portugal
}

Cite as: van Zeller M, Drummond M. Pneumology and sleep disordered breathing training in Portugal. Breathe 2018; 14: 248-249.
In Portugal, a medical degree takes 6 years, including a master's degree, ever since the Bologna Agreement of 2009. After this, and according to the Ordem dos Médicos (College of Physicians), one must complete 2 years of fully supervised clinical practice to be able to practice medicine. During this period, junior doctors rotate through several different medical and chirurgical specialties, after which and according to their grade on a national examination, they chose a specialty and location. Pneumology/respiratory medicine is one of the options and every year roughly 30 residents begin this 5 -year specialty. Junior doctors usually choose where to perform their residency according to the subspecialties available in a particular hospital, their relationship with academia, opportunities for research projects and to go abroad, among other factors.

The training programme is mandatory for all residents; the basic criteria to become a respiratory specialist are thus well defined. Likewise, the recommended number of posters, oral presentations and publications for the 5 years of residency is pre-determined.

I completed my residency at Centro Hospitalar de São João (figure 1) in Porto (figure 2), which is a university hospital with all subspecialties available. This hospital and the Faculty of Medicine of the University of Porto share facilities and have a collaboration protocol.

When I was resident, it was compulsory to complete 12 months of internal medicine, 4 months of intensive care, 18 months of general respiratory practice, 6 months of bronchoscopy, 3 months of lung function, oncology, pulmonary rehabilitation and allergology, and 2 months of cardiothoracic surgery and tuberculosis and non-tuberculosis mycobacteria in primary care. I was left with 4 months of optional respiratory themes; I chose chest imaging and respiratory infection.

The Portuguese authorities support and promote internships outside the country, if added value is expected. The Portuguese Respiratory Society (SPP) president at the time instituted a grant for interns; therefore, after a successful application I had the opportunity and honour to be part of Robert Wilson's team at the Royal Brompton Hospital, London, UK. This was a life-changing opportunity, even down to the smallest details. The relevance of networking was astonishing. Through this internship, I was introduced to the European Respiratory Society (ERS) Infectious Disease Assembly and its members. Afterwards, I was invited to become the Portuguese Junior ERS Delegate for SPP.

Although the head of department assigns a tutor to each intern, since the respiratory areas are so different and the opportunities so

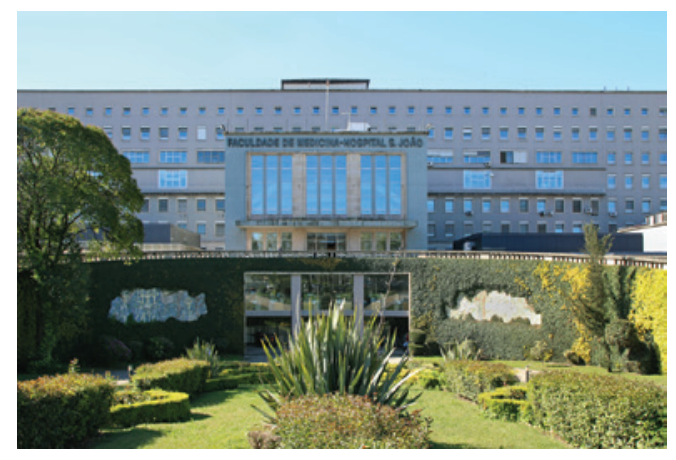

Figure 1 Centro Hospitalar de São João, Porto, Portugal. 


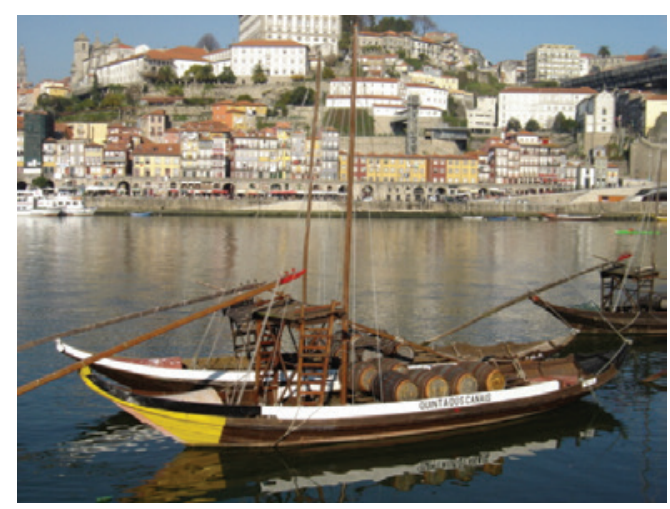

Figure 2 The city of Porto, Portugal.

various, it is crucial to integrate "micro" medical research networks that are vital to the sharing and dissemination of knowledge and learning resources [1]. Opportunities are increasing, and it is up to each one of us to seize them.

At the time of my internship (2009-2013), there was no item for training in sleep disordered breathing in the curriculum, although it was already a well-recognised health problem. Being a huge part of a respiratory physician's clinical practice and contemplating a career in that field in that university hospital, this was an extra part of my training.

The Portuguese respiratory training curriculum was updated in 2016 [2], considering the impact of sleep medicine in clinical practice. Hence, a 3-month period exclusively dedicated to the diagnosis and treatment of sleep breathing disorders, with particular dedication to obstructive sleep apnoea, was included.

Since junior doctors may choose where they want to perform their 3-month slot, usually sleep laboratories covering more fields are more desirable, as are those laboratories active in research projects since research in this area is much encouraged. The Sleep Laboratory at Centro Hospitalar de São João has expertise in diagnosis (performing level 1 and 3 polysomnography, multiple sleep latency tests, and maintenance wakefulness tests), treatment and follow-up of patients with sleep breathing disorders. The team is multidisciplinary, including respiratory physicians, cardiorespiratory and sleep technicians, respiratory physiotherapists, nutritionists, and a psychologist, and works in collaboration with other specialties, including otorhinolaryngology, oral and maxillofacial surgery, and neurology, among others. The laboratory has recently joined the European Sleep Apnoea Database (ESADA) [3].

In our laboratory, the number of examinations junior doctors need to review to be considered apt has previously been determined, as well as the number of first medical appointments and follow-ups that should be performed. Additionally, in Portugal, the College of Physicians has a sleep medicine certification, for which doctors from several medical specialties, including respiratory, neurology and psychiatry, may apply.

Looking back, all those experiences had a significant impact; exposing oneself to the world and to different realities is a fast but enriching way to grow. Years pass and interests change but friends, medical and research networks, and knowledge last forever.

In fact, after experiencing other countries' systems, I must say that the Portuguese respiratory internship is very well organised and friendly. It is not perfect, with room for improvement, as is the case for almost everything, but it compulsorily covers all the essential respiratory areas and if one yearns for more, there are ways to accommodate this.

Moreover, the ERS and the SPP are now working together on ERS curriculum development strategies, HERMES and Summer School initiatives.

As a famous Portuguese poet, referring to the Portuguese maritime discoveries, would say, "Worth it? It's all worth it, If the soul is not small” [4].

\section{Conflict of interest}

None declared.

\section{References}

1. Riha RL. Breathe: global networks in respiratory disease. Breathe 2017; 13: 161-162.

2. Diário da República n. 249/2016, Série I de 2016-12-29.

3. Hedner J, Grote L, Bonsignore $M$, et al. The
European Sleep Apnoea Database (ESADA): report from 22 European sleep laboratories. Eur Respir J 2011; 38: 635-642.

4. Fernando Pessoa. Lisboa: Parceria A.M. Pereira, 1934. - 100, [2] p.; $19 \mathrm{~cm}$. 